\title{
Avaliação de qualidade de vida em portadores de blefaroptose
}

\author{
Quality oflife assessment in patients with blepharoptosis
}

\author{
Márcia Emiko Asano ${ }^{1}$ \\ Midori Hentona Osaki ${ }^{2}$
}

Trabalho de conclusão do curso de MBA em Saúde e Administração da Prática Oftalmológica do Departamento de Oftalmologia da Universidade Federal de São Paulo - UNIFESP - São Paulo (SP) - Brasil.

${ }^{1}$ Estagiária do Setor de Cirurgia Plástica Ocular da Universidade Federal de São Paulo - UNIFESP - São Paulo (SP) - Brasil e aluna do curso de "Master in Business Administration" (MBA) em Saúde e em Administração da Prática Oftalmológica da UNIFESP - São Paulo (SP) - Brasil.

2 Chefe do Setor de Cirurgia Plástica Ocular da UNIFESP - São Paulo (SP) - Brasil.

Endereço para correspondência: Márcia Emiko Asano. Rua Martiniano de Carvalho, 611 - Apto. 51 - São Paulo (SP) CEP 01321-001

E-mail: marciaasano@uol.com.br

Recebido para publicação em 12.10.2005

Versão revisada recebida em 11.12.2005

Aprovação em 12.01.2006

\section{RESUMO}

Objetivo: Avaliar a qualidade de vida dos portadores de blefaroptose e o impacto da correção cirúrgica sobre os mesmos. Métodos: Foram aplicados questionários adaptados do "Visual Function Questionnaire" (VFQ) antes e após tratamento cirúrgico em 21 pacientes com blefaroptose congênita ou adquirida. Resultados: A pontuação média dos pacientes, no pré-operatório, foi de 74 e, no pós-operatório, 81 e diferença pré e póscirurgia estatisticamente significante de $7(\mathrm{p}=0,004)$. Na avaliação das questões $(\alpha=0,002)$ e subdomínios $(\alpha=0,004)$ houve diferença estatisticamente significante apenas para o subdomínio saúde mental $(\mathrm{p}=0,001)$. Conclusão: A qualidade de vida dos pacientes com blefaroptose apresentou melhora significante após a intervenção cirúrgica na avaliação com o VFQ. Os resultados reforçam a necessidade do uso de questionários elaborados especificamente para esta doença.

Descritores: Qualidade de vida; Blefaroptose/cirurgia; Questionário

\section{INTRODUÇ̃̃O}

Blefaroptose é a condição em que a margem da pálpebra superior (PS) encontra-se posicionada em um nível mais baixo que o normal, na posição primária do olhar. A ptose usualmente representa um problema estético, porém pode levar a um sério déficit funcional nos casos em que cobre o eixo visual $^{(1-2)}$.

A ptose palpebral pode ser congênita ou adquirida e o tratamento geralmente é cirúrgico. No entanto, certos casos devem ser tratados clinicamente e eventualmente pode estar contra-indicada qualquer medida terapêutica ${ }^{(1-3)}$.

Não há dados suficientes disponíveis a respeito da incidência de ptose palpebral tanto internacionalmente quanto para um determinado país. Há igual freqüência entre diferentes raças e entre os $\operatorname{sexos}^{(3)}$.

A Organização Mundial da Saúde (OMS) define qualidade de vida como uma percepção individual de sua posição na vida, no contexto cultural e no sistema de valores em que se vive e em relação aos seus objetivos, expectativas, padrões e interesses, e saúde como um estado de completo bem-estar físico, mental e social e não meramente a ausência de doença ou enfermidade $^{(4-5)}$.

Estudos prévios têm demonstrado que os efeitos deletérios das doenças oculares são responsáveis pela redução da qualidade de vida relacionada à saúde. Embora, a blefaroptose esteja associada à perda de campo visual superior, poucos trabalhos têm sido desenvolvidos a respeito da blefaroptose e o impacto da cirurgia de correção da mesma no estado funcional e qualidade de vida destes pacientes ${ }^{(6-8)}$. 
Há diversos instrumentos para avaliar a qualidade de vida, cuja validade e confiabilidade já foram demonstrados em populações diferentes. Estes servem para explorar os impactos específicos do déficit visual como também, comparar diferentes terapêuticas em relação ao risco-benefício ${ }^{(4-20)}$.

Vários modelos de avaliação de qualidade de vida são descritos na literatura e se subdividem em gerais e específicos. Os questionários gerais são utilizados para avaliar a qualidade de vida como um todo sem se restringir às peculiaridades de uma determinada doença, como o "Sickness Impact Profile" (SIP), o "Short Form 36" (SF-36), o "Duke-UNC Health Profile" (DUHP), o "Comprehensive Assessment and Referral Evaluation" (CARE). E os específicos focalizam as características de uma doença em particular e são mais adequados para avaliar os efeitos de uma intervenção terapêutica específica. O "Vision Function Questionnaire" (VFQ), o "Activities of daily vision scale" (ADVS), o VF-14 (14-Item Visual Functioning Index), entre outros foram desenvolvidos para avaliação da qualidade de vida em doenças oculares ${ }^{(4-20)}$.

O VFQ é um questionário derivado do "Vision Activities Questionnaire" (VAQ) e avalia tanto qualidade de vida, quanto função visual, subjetivamente ${ }^{(9-10)}$.

O objetivo deste estudo foi avaliar a qualidade de vida dos portadores de blefaroptose e o impacto da correção cirúrgica sobre os mesmos, através de um questionário elaborado para avaliação da qualidade de vida em doenças oculares.

\section{MÉTODOS}

Vinte e um pacientes com blefaroptose congênita ou adquirida, atendidos no ambulatório de Cirurgia Plástica Ocular do Departamento de Oftalmologia da UNIFESP/EPM, no período de março/2004 a junho/2005, submetidos a tratamento cirúrgico, participaram deste estudo após termo de consentimento. Os critérios de inclusão foram os seguintes: idade de 18 anos ou mais; habilidade para completar questionário pré e pós-operatório; acuidade visual corrigida de 20/50 no olho acometido; ptose com distância margem-reflexo (DMR) de $2 \mathrm{~mm}$ ou menos.

Utilizou-se um questionário adaptado do $\mathrm{VFQ}^{(9)}$ para avaliação da qualidade de vida, que foi aplicado antes e após a cirurgia (4-8 semanas), por meio de entrevista pessoal realizada por dois oftalmologistas.

O VFQ contém 25 questões agrupados em 13 subdomínios $^{(9)}$, com uma ou mais questões em cada subdomínio. Cada questão apresenta cinco opções de resposta, sendo cada resposta submetida a uma pontuação que varia de 0 a 100 . A pontuação final é obtida calculando-se a média das pontuações das respostas, alcançando-se um escore para cada paciente, cujo valor mínimo é 0 e o valor máximo é 100 . Quanto maior o escore alcançado, melhor a qualidade de vida e a função visual do paciente.

Os dados foram submetidos à análise estatística, através dos testes t de Student para escore geral e teste de Wilcoxon para avaliar as questões e subdomínios.

\section{RESULTADOS}

Dos 21 pacientes avaliados, $14(66,7 \%)$ eram do sexo feminino e $7(33,3 \%)$ do sexo masculino. A idade média foi de 57 anos, variando de 21 a 80 anos. Dois pacientes $(9,5 \%)$ apresentavam ptose congênita e o restante, adquirida.

A pontuação média dos pacientes, no pré-operatório, foi de 74 e, no pós-operatório, 81 (Tabela 1A). Encontrou-se uma diferença média de 7 no escore geral com limites de intervalo de confiança de 95\% iguais a 3 e 12 (Tabela 1B e Gráfico 1).

\begin{tabular}{|lccc|}
\hline \multicolumn{4}{c}{ Tabela 1A. Medidas-resumo do escore geral } \\
\cline { 2 - 4 } Medidas-resumo & \multicolumn{2}{c|}{ Escore total } & $\begin{array}{c}\text { Diferença } \\
\text { pós - pré }\end{array}$ \\
\cline { 2 - 4 } $\mathrm{N}$ & $\begin{array}{c}\text { Pré } \\
\text { Média }\end{array}$ & Pós & 21 \\
Desvio-padrão & 74 & 81 & 7 \\
Mediana & 23 & 20 & 10 \\
Mínimo & 87 & 91 & 7 \\
Máximo & 23 & 23 & -12 \\
\hline
\end{tabular}

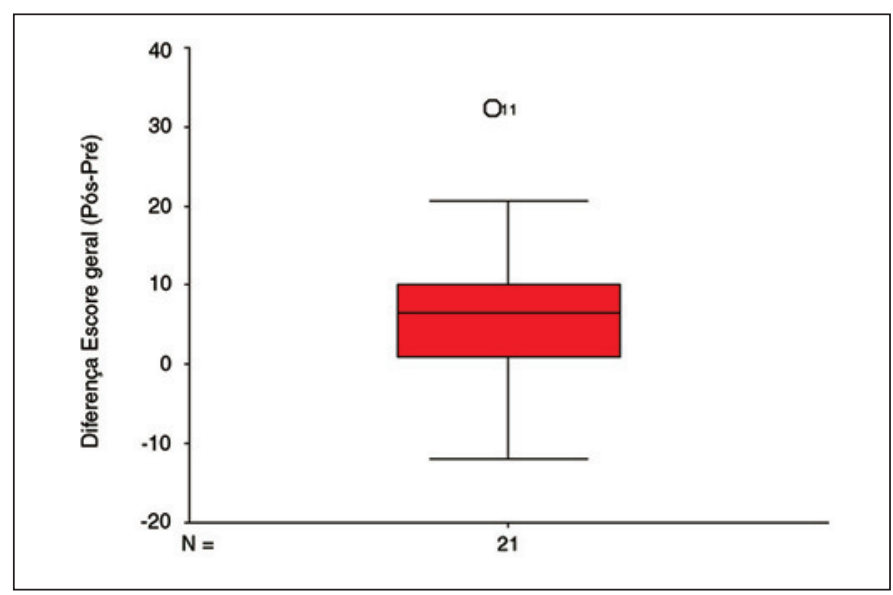

Gráfico 1 - "Box plot" da diferença (pós - pré) do escore geral

\begin{tabular}{|c|c|c|c|c|c|}
\hline \multicolumn{6}{|c|}{ Tabela 1B. Teste $t$ de Student pareado } \\
\hline \multirow{2}{*}{$\begin{array}{c}\text { Diferença Média } \\
\text { pós - pré }\end{array}$} & \multirow{2}{*}{ Desvio-padrão } & \multirow{2}{*}{ Erro-padrão } & \multicolumn{2}{|c|}{ Intervalo de confiança (95\%) } & \multirow[t]{2}{*}{$\mathbf{p}$} \\
\hline & & & Limite inferior & Limite superior & \\
\hline 7 & 10 & 2 & 3 & 12 & 0,004 \\
\hline
\end{tabular}


Na comparação das questões pré e pós, sem considerar nenhum tipo de correção estatística $(\alpha=0,05)$, é possível dizer que foram encontradas diferenças para as questões $(2,3,18$, 21, 22 e 24) segundo tabela 2.

Sendo que em todos os casos houve melhora. Entretanto, considerando a correção de Bonferroni, ou seja, admitindo um

\begin{tabular}{|c|c|c|c|c|c|}
\hline \multirow[t]{2}{*}{ Questões } & \multicolumn{2}{|c|}{$Q$ pós - $Q$ pré } & \multirow[t]{2}{*}{ Empates } & \multirow[t]{2}{*}{ Total } & \multirow[t]{2}{*}{$p$} \\
\hline & $\begin{array}{l}\text { Postos } \\
\text { positivos }\end{array}$ & $\begin{array}{c}\text { Postos } \\
\text { negativos }\end{array}$ & & & \\
\hline Q1 & 5 & 4 & 12 & 21 & $>0,999$ \\
\hline Q2 & 7 & 1 & 13 & 21 & $0,023^{*}$ \\
\hline Q3 & 14 & 3 & 4 & 21 & $0,007^{*}$ \\
\hline Q4 & 5 & 5 & 11 & 21 & 0,832 \\
\hline Q5 & 4 & 1 & 15 & 20 & 0,098 \\
\hline Q6 & 5 & 4 & 12 & 21 & 0,755 \\
\hline Q7 & 1 & 4 & 16 & 21 & 0,157 \\
\hline Q8 & 5 & 2 & 14 & 21 & 0,438 \\
\hline Q9 & 5 & 2 & 14 & 21 & 0,340 \\
\hline Q10 & 3 & 1 & 17 & 21 & 0,450 \\
\hline Q11 & 0 & 0 & 21 & 21 & $>0,999$ \\
\hline Q12 & 3 & 1 & 17 & 21 & 0,317 \\
\hline Q13 & 3 & 0 & 17 & 20 & 0,102 \\
\hline Q14 & 8 & 3 & 10 & 21 & 0,120 \\
\hline Q15c & 0 & 1 & 6 & 7 & 0,317 \\
\hline Q16 & 0 & 1 & 5 & 6 & 0,317 \\
\hline Q17 & 6 & 2 & 13 & 21 & 0,155 \\
\hline Q18 & 6 & 0 & 15 & 21 & $0,026^{*}$ \\
\hline Q19 & 0 & 3 & 18 & 21 & 0,109 \\
\hline Q20 & 5 & 1 & 15 & 21 & 0,114 \\
\hline Q21 & 8 & 0 & 13 & 21 & $0,010^{*}$ \\
\hline Q22 & 6 & 1 & 14 & 21 & $0,033^{*}$ \\
\hline Q23 & 3 & 2 & 16 & 21 & 0,496 \\
\hline Q24 & 5 & 0 & 16 & 21 & $0,039^{*}$ \\
\hline Q25 & 3 & 0 & 18 & 21 & 0,109 \\
\hline
\end{tabular}

nível de significância $\alpha=0,002$, não é possível afirmar que existe diferença estatisticamente significante para nenhuma questão.

Quanto aos subdomínios (Tabela 3 e Gráfico 2), sem considerar nenhum tipo de correção $(\alpha=0,05)$, observou-se diferenças estatisticamente significantes para os subdomínios: visão, atividades para longe, saúde mental, atividades diárias e dependência.

Entretanto, considerando a correção de Bonferroni $(\alpha=0,004)$, poderia se afirmar que houve diferença estatisticamente significante apenas para o subdomínio da Saúde mental.

\section{DISCUSSÃO}

Na oftalmologia, tradicionalmente depende-se de medidas psicofísicas objetivas para avaliar a capacidade funcional do paciente. Medidas de acuidade visual e campo visual particularmente são usados clinicamente para julgar a eficácia do tratamento. Embora, a mensuração objetiva da piora visual,

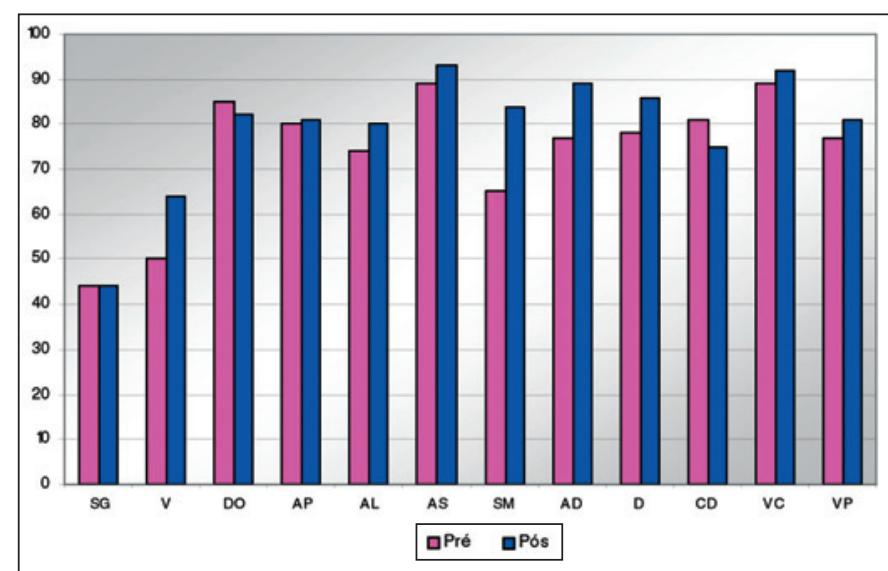

$\mathrm{SG}=$ saúde geral; $\mathrm{V}=$ visão; $\mathrm{DO}=$ dor ocular; $\mathrm{AP}=$ atividades para perto; $\mathrm{AL}=$ atividades para longe; $A S=$ aspectos sociais; $S M=$ saúde mental; $A D=$ atividades diárias; $D=$ dependência; $\mathrm{CD}=$ capacidade de dirigir; $\mathrm{VC}=$ visão de cores; $\mathrm{VP}=$ visão periférica.

Gráfico 2 - Comparação dos escores médios dos subdomínios do VFQ, pré e pós-tratamento de blefaroptose

\begin{tabular}{|c|c|c|c|c|c|c|}
\hline Subdomínios & Postos positivos & Postos & negativos & Empates & Total & p \\
\hline Saúde geral (SG) & 5 & & 4 & 12 & 21 & $>0,999$ \\
\hline Visão (V) & 7 & & 1 & 13 & 21 & $0,023^{*}$ \\
\hline Dor ocular (DO) & 5 & & 6 & 10 & 21 & 0,470 \\
\hline Atividades para perto (AP) & 5 & & 2 & 13 & 20 & 0,605 \\
\hline Atividades para longe $(A L)$ & 13 & & 1 & 7 & 21 & $0,015^{\star}$ \\
\hline Aspectos sociais (AS) & 3 & & 0 & 17 & 20 & 0,102 \\
\hline Saúde mental (SM) & 16 & & 2 & 3 & 21 & $0,001^{* *}$ \\
\hline Atividades diárias (AD) & 7 & & 2 & 12 & 21 & $0,028^{*}$ \\
\hline Dependência (D) & 9 & & 1 & 11 & 21 & $0,011^{*}$ \\
\hline Capacidade de dirigir (CD) & 0 & & 1 & 5 & 6 & 0,317 \\
\hline Visão de cores (VC) & 3 & & 1 & 17 & 21 & 0,317 \\
\hline Visão periférica (VP) & 3 & & 1 & 17 & 21 & 0,450 \\
\hline
\end{tabular}


ainda seja necessária, está longe de ser considerada suficiente. Atualmente, há um crescimento da demanda para inclusão de avaliações subjetivas da função visual na mensuração dos resultados do tratamento ${ }^{(10)}$.

A maioria dos instrumentos de avaliação de qualidade de vida baseada na função visual foram inicialmente utilizados em pacientes com catarata ${ }^{(9-10,17,19)}$. Ao avaliar portadores de blefaroptose, através do VFQ, o escore geral mostrou uma melhora da qualidade de vida dos pacientes. Em relação aos subdomínios, utilizando a correção estatística $(\alpha=0,004)$, observou-se diferença estatisticamente significante apenas no subdomínio Saúde mental.

Embora, a correção da blefaroptose seja um procedimento funcional, é também cosmético ${ }^{(1,3)}$. Cirurgias cosméticas têm mostrado beneficiar tanto no aspecto psicológico ${ }^{(8,20)}$ quanto no estilo de vida dos pacientes, aliviando sintomas de depressão e melhorando a qualidade de vida e problemas com a imagem corporal $^{(20)}$, justificando assim a alteração significante no subdomínio Saúde mental do questionário adaptado do VFQ para doenças oculares em geral. Porém sem correção estatística $(\alpha=0,05)$, cinco subdomínios (Visão, Atividades para longe, Saúde mental, Atividades diárias e Dependência) apresentaram diferença estatisticamente significante.

Federici et al. encontraram uma pontuação média de 52 no pré e 82 no pós-operatório( ${ }^{(7)}$, utilizando um questionário de 27 itens, elaborado para avaliar o efeito da posição palpebral na qualidade de vida e função visual dos pacientes, cujas questões foram adaptadas do $\operatorname{SIP}^{(12)}$, $\mathrm{CARE}^{(13)}$ e DUHP(14), focalizando em atividades e sintomas que potencialmente possam ser afetados por prejuízo visual tipicamente associado à ptose palpebral $^{(6-7,15)}$. Em nosso estudo, a pontuação média, no préoperatório, foi de 74 e, no pós, de 81 . Apesar da diferença de pontuação entre pré e pós nos dois estudos, ambos demonstraram uma melhora na qualidade de vida destes pacientes. Esta diferença pode ser explicada pelo fato dos instrumentos desenvolvidos especificamente para uma doença tenderem a ser mais sensíveis na detecção de efeitos do tratamento do que os genéricos destinados a avaliar a qualidade de vida relacionada à saúde em geral ${ }^{(5,17)}$.

Analisando-se as questões, com $\alpha=0,002$, não se pode afirmar a existência de diferença estatisticamente significante entre as questões pré e pós-operatório no presente estudo. Enquanto, Federici et al. ${ }^{(7)}$ mostraram uma melhora pós-cirúrgica significante para todas as questões, exceto para a questão 12 (outras) e para 25 (saúde geral). Battu et al. ${ }^{(6)}$ utilizando o mesmo questionário que Federici et al. ${ }^{(7)}$ em estudo preliminar, encontraram maiores alterações para as seguintes atividades (em ordem decrescente): ler, habilidade para realizar trabalhos manuais finos, assistir TV, ler sinais na estrada ou ver semáforos enquanto dirige. Itens mais genéricos (bem-estar geral, situação econômica, nível de energia, nível geral de saúde, andar sem assistência) não demonstraram alterações significantes. Estes podem não ser as características mais indicadas para se monitorizar o sucesso ou falência do reparo da blefaroptose.
Pacientes com blefaroespasmo essencial (BE) e espasmo hemifacial $(\mathrm{EH})$ apresentaram melhora da qualidade de vida após tratamento com toxina botulínica tipo A (BTX-A) em estudo utilizando o SF-36, o qual evidenciou uma piora da saúde mental, bem como no presente estudo, além de limitações físicas e emocionais ${ }^{(11)}$.

$\mathrm{Na}$ literatura, vários estudos têm demonstrado melhora na qualidade de vida referente à saúde após cirurgia de catara$\operatorname{ta}^{(9-10,17,19)}$. Cassard et al. apresentaram uma pontuação média de 76,5 no pré e 93,2 no pós-operatório, na avaliação de pacientes com catarata ${ }^{(17)}$, usando o VF-14, desenvolvido por Steinberg et al. especificamente para pacientes com catara$\mathrm{ta}^{(10)}$. A nossa pesquisa encontrou pontuação (74) semelhante antes do tratamento. Assim, apesar de serem patologias oculares diferentes, a catarata levando à perda visual primariamente central e a blefaroptose a um prejuízo de campo visual superior $^{(6-8)}$, ambas apresentam uma redução no estado funcional e qualidade de vida associados à saúde e melhora após tratamento. A pontuação média mais elevada após a cirurgia de catarata provavelmente se deve aos resultados geralmente mais rápidos, que aparecem até mesmo horas após o tratamento ${ }^{(18)}$, além da catarata normalmente ser mais incapacitante, dependendo da severidade. A blefaroptose, embora muitas vezes ocasione um déficit funcional importante ao cobrir o eixo visual, representa, na maioria das vezes, um problema cosmético. Além disso, o paciente pode usar artifícios ou medidas para melhorar esta incapacidade, como manter posição de cabeça com queixo elevado, usar o músculo frontal ou os dedos para elevar a pálpebra ${ }^{(1-3)}$.

Estudos com o VF-14, envolvendo pacientes transplantados de córnea, observaram pontuação média variando de 68,3 a $77^{(18-19)}$. Entretanto, estes não avaliaram os pacientes antes do transplante de córnea, não conhecendo assim a diferença entre pré e pós-cirurgia. Esses valores mais baixos pós-transplante de córnea em relação aos pacientes pseudofácicos e pós-correção de blefaroptose podem ser explicados pelo resultado visual lento, recuperação cirúrgica prolongada e complicações pós-cirúrgica mais freqüentes ${ }^{(18)}$. Musch et al. observaram maiores problemas com os aspectos sociais e físicos nos indivíduos com acuidade visual relativamente pobre na aplicação do SF-36 ${ }^{(19)}$.

Em geral, os vários instrumentos utilizados para avaliar a qualidade de vida em relação à função visual das inúmeras patologias têm mostrado uma melhora após tratamento ${ }^{(6-8,10-11,17,19)}$.

\section{CONCLUSÃO}

A qualidade de vida dos pacientes com blefaroptose apresentou uma melhora significante após a intervenção cirúrgica, quando comparado o escore geral. Porém no pareamento de questões e subdomínios, o único resultado estatisticamente significante (utilizando o nível de significância corrigido) foi o subdomínio Saúde mental. 
Estes resultados reforçam a necessidade do uso de questionários elaborados especificamente para a patologia em estudo, a fim de alcançar uma melhor avaliação da qualidade de vida e impacto do tratamento dos pacientes com blefaroptose.

\section{AGRADECIMENTOS}

À Dra. Milena Atique-Tacla pelo auxílio nas cirurgias e à Dra. Patrícia Yuri Miyasato pela ajuda na aplicação dos questionários.

\section{ABSTRACT}

Purpose: To assess the health-related quality of life in blepharoptosis patients and the impact after surgical management on their quality of life. Methods: Twenty-one patients with congenital or acquired blepharoptosis completed a questionnaire which was adapted from the Visual Function Questionnaire (VFQ), before and after surgical management. Results: Mean preoperative score was 74 and mean postoperative score was 81 and there was a statistically significant difference between mean pre- and postoperative scores of $7(\mathrm{p}=0.004)$. There was a statistically significant difference only for the mental health subdomain $(\mathrm{p}=0.001)$ in the evaluation of the questions $(\alpha=0.002)$ and subdomains $(\alpha=0.004)$. Conclusion: The quality of life in blepharoptosis patients was significantly better after surgical management in the assessment using the VFQ. The outcomes emphasize the need for of using questionnaires designed specifically for this disease.

Keywords: Quality of life; Blepharoptosis/surgery; Questionnaire

\section{REFERÊNCIAS}

1. Nesi FA, Lisman RD, Levine MR, editors. Smith's ophthalmic plastic and reconstructive surgery. Saint Louis: Mosby; 1998.

2. Soares EJC, Figueiredo ARP, Souza GL, Almeida HC, Oliveira LRM, Magalhães
MM, Portellinha WM. Blefaroptose. In: Soares EJC, Moura EM, Gonçalves JOR, editors. Cirurgia plástica ocular. São Paulo: Rocca; 1997. p.77-152.

3. Finsterer J. Ptosis: causes, presentation, and management. Aesthetic Plast Surg. 2003;27(3):193-204. Epub 2003 Aug 21. Review.

4. Wolffsohn JS, Cochrane AL, Watt NA. Implementation methods for vision related quality of life questionnaires. Br J Ophthalmol. 2000;84(9):1035-40.

5. Testa MA, Simonson DC. Assessment of quality-of-life outcomes. N Engl J Med. 1996;334(13):835-40.

6. Battu VK, Meyer DR, Wobig JL. Improvement in subjective visual function and quality of life outcome measures after blepharoptosis surgery. Am J Ophthalmol. 1996;121(6):677-86.

7. Federici TJ, Meyer DR, Lininger LL. Correlation of the vision-related functional impairment associated with blepharoptosis and the impact of blepharoptosis surgery. Ophthalmology. 1999;106(9):1705-12.

8. Warwar RE, Bullock JD, Markert RJ, Marciniszyn SL, Bienenfeld DG. Social implications of blepharoptosis and dermatochalasis. Ophthal Plast Reconstr Surg. 2001;17(4):234-40.

9. Ferraz EVAP, Lima CA, Cella W, Arieta CEL. Adaptação de questionário de avaliação da qualidade de vida para aplicação em portadores de catarata. Arq Bras Oftalmol. 2002;65(3):293-8.

10. Massof RW, Rubin GS. Visual function assessment questionnaires. Surv Ophthalmol. 2001;45(6):531-48. Review.

11. Osaki MH, Belfort Júnior R. Qualidade de vida e custos diretos em pacientes com blefaroespasmo essencial e espasmo hemifacial, tratados com toxina botulínica-A. Arq Bras Oftalmol. 2004;67(1):43-9.

12. Bergner M, Bobbitt RA, Carter WB, Gilson BS. The Sickness Impact Profile: development and final revision of a health status measure. Med Care. 1981;19 (8):787-805

13. Golden RR, Teresi JA, Gurland BJ. Development of indicator scales for the Comprehensive Assessment and Referral Evaluation (CARE) interview schedule. J Gerontol. 1984;39(2):138-46.

14. Parkerson GR Jr, Gehlbach SH, Wagner EH, James SA, Clapp NE, Muhlbaier LH. The Duke-UNC Health Profile: an adult health status instrument for primary care. Med Care. 1981;19(8):806-28.

15. Brémond-Gignac D, Tixier J, Missotten T, Laroche L, Beresniak A. Évaluation de la qualité de vie en ophtalmologie. Presse Med. 2002;31(34):1607-12.

16. Scott IU, Schein OD, West S, Bandeen-Roche K, Enger C, Folstein MF. Functional status and quality of life measurement among ophthalmic patients. Arch Ophthalmol. 1994;112(3):329-35.

17. Cassard SD, Patrick DL, Damiano AM, Legro MW, Tielsch JM, Diener-West $\mathrm{M}$, et al. Reproducibility and responsiveness of the VF-14. An index of functional impairment in patients with cataracts. Arch Ophthalmol. 1995;113(12): 1508-13.

18. Atique D, Goulart DG, Lake JC, Lima FA, Felberg S, Nishiwaki-Dantas MC. Qualidade de vida após transplante penetrante de córnea. Arq Bras Oftalmol. 2002;65(3):351-4.

19. Musch DC, Farjo AA, Meyer RF, Waldo MN, Janz NK. Assessment of healthrelated quality of life after corneal transplantation. Am J Ophthalmol. 1997;124 (1): $1-8$.

20. Mauriello JA Jr. Perspective: cosmetic eyelid surgery from the patient's perspective. Ophthal Plast Reconstr Surg. 2003;19(4):320-2. 